\title{
Effects of Viscous Shear Stresses on the Squeeze Films of Porous Parallel Rectangular Plates
}

Jaw-Ren Lin

Professor, Department of Mechanical Engineering, Nanya Institute of Technology P.O. Box 324-2259, Chung-Li, Taiwan 320, R.O.C., jrlin@nanya.edu.tw

Long-Jin Liang

Assistant Professor, Department of Mechanical Engineering, Nanya Institute of Technology, P.O. Box 324-2259, Chung-Li, Taiwan 320, R.O.C.

Rong-Fang Lu

Assistant Professor, Department of Mechanical Engineering, Nanya Institute of Technology, P.O. Box 324-2259, Chung-Li, Taiwan 320, R.O.C.

Follow this and additional works at: https://jmstt.ntou.edu.tw/journal

Part of the Mechanical Engineering Commons

\section{Recommended Citation}

Lin, Jaw-Ren; Liang, Long-Jin; and Lu, Rong-Fang (2004) "Effects of Viscous Shear Stresses on the Squeeze Films of Porous Parallel Rectangular Plates," Journal of Marine Science and Technology: Vol. 12: Iss. 2, Article 6.

DOI: $10.51400 / 2709-6998.2227$

Available at: https://jmstt.ntou.edu.tw/journal/vol12/iss2/6

This Research Article is brought to you for free and open access by Journal of Marine Science and Technology. It has been accepted for inclusion in Journal of Marine Science and Technology by an authorized editor of Journal of Marine Science and Technology. 
Effects of Viscous Shear Stresses on the Squeeze Films of Porous Parallel Rectangular Plates

Acknowledgements

This work was supported by the National Science Council of ROC: NSC 90-2212-E-253-004.

This research article is available in Journal of Marine Science and Technology: https://jmstt.ntou.edu.tw/journal/ 


\title{
EFFECTS OF VISCOUS SHEAR STRESSES ON THE SQUEEZE FILMS OF POROUS PARALLEL RECTANGULAR PLATES
}

\author{
Jaw-Ren Lin*, Long-Jin Liang **, and Rong-Fang Lu**
}

Key words: squeeze films, proous plates, Brinkman model, rectangular plates.

\section{ABSTRACT}

Considering the effects of viscous shear stresses, a study of squeeze-film behavior between porous parallel plates with finite width is presented. Based upon the Brinkman model (BM), two general coupled Reynolds equations derived for curved surfaces [20] are applied to predict the two-dimensional squeeze-film pressure and thereafter the squeeze-film characteristics. The results show that the BM predicts quite different squeeze-film behaviors to those obtained by using the slip-flow model (SFM) and the Darcy model (DM). Comparing with the SFM, the viscous shear effects provide an increase in the load-carrying capacity and lengthen the response time of finite porous squeeze-film plates. However, these trends are reversed as compared to the DM. On the whole, the effects of viscous shear stresses of the BM upon the load-carrying capacity and the response time are more pronounced for the plates tending to be wide.

\section{INTRODUCTION}

The investigation of squeeze-film characteristics between porous surfaces plays an important role in engineering and industrial applications. To describe the fluid motion through a porous matrix three models have been used in the studies of hydrodynamic porous bearings: the Darcy model (DM), the slip-flow model (SFM) and the Brinkman model (BM). In the DM, Darcy's law was adopted to govern the fluid flow in the porous matrix and the no-slip condition was utilized at the film/porous surface interface. In the SFM, Darcy's law was used to describe the fluid flow in the porous matrix but a tangential slip velocity is be taken into consideration at the fluid/porous matrix interface by Beavers and Joseph [1] and Beavers et al. [2]. Since the artificial SFM takes into account the phenomena of slip velocity at the film/porous matrix interface, an incom-

Paper Submitted 01/06/04, Accepted 04/29/04. Author for Correspondence: Jaw-Ren Lin.E-mail: jrlin@nanya.edu.tw.

*Professor, Department of Mechanical Engineering, Nanya Institute of Technology P.O. Box 324-2259, Chung-Li, Taiwan 320, R.O.C.

**Assistant Professor, Department of Mechanical Engineering, Nanya Institute of Technology, P.O. Box 324-2259, Chung-Li, Taiwan 320, R.O.C. patible discontinuity of tangential velocity component occurs across the interface. According to the discussion of Neale and Nader [14] in coupled flows within a channel flow and a bounding porous medium, a viscous shear term in the Brinkman equations matching the stress across the interface is physically compatible with the continuous velocity profile within the porous matrix. Therefore in the BM, the Brinkman equations are adopted to govern the fluid flow in the porous matrix and the continuity conditions of velocities and shear stresses are applied at the film/porous surface interface. In the conventional study of porous squeeze-film surfaces using the DM, porous squeeze-film behaviors are presented for journal bearings [3, 15], spherical bearings [10], circular disks [11, 12], annular disks [20, 21], and rectangular plates [18]. According to the SFM, the effects of slip velocity on porous squeeze-film characteristics are analyzed in journal bearings [13], circular disks [9], annular disks [17], rectangular plates [19], and plates with different shapes [16]. Applying the BM, the viscous shear effects upon porous squeeze-film characteristics were presented for journal bearings [6], spherical bearings [8], and circular disks [7]. In a recent study [5], the BM is applied to derive the general porous squeeze-film Reynolds equations and to investigate the squeeze-film behavior between infinite-width parallel plates. Since the squeeze film motion of finite plates may often occur in practical application, a further extension is needed.

The present study is concerned mainly with the effects of viscous shear stresses in the squeeze-film behavior between porous parallel rectangular plates. Based upon the Brinkman model, two coupled Reynolds equations governing the squeeze-film pressure are solved numerically. Compared with the Darcy model and slipflow model, the squeeze film characteristics are presented for different values of permeability parameter and length-to-width ratio.

\section{ANALYSIS}

Fig. 1 shows the squeeze-film geometry between 
porous parallel rectangular plates of length $L$ and width $D$ at the section plane of $y=0$. The lower plate with a porous facing of thickness $H$ is fixed, and the upper plate has a squeezing velocity $d h / d t$. The lubricant is taken to be an incompressible Newtonian fluid having constant properties. In addition to the conventional thin-film lubrication assumptions, it is assumed that the porous material is homogeneous and isotropic, the flow in the porous matrix is laminar, and the pressure gradient across the porous medium is a unknown function $\mathrm{g}$ $(x, y)$. According to Lin et al. [5], the two coupled Reynolds equations governing the film pressure $p$ and the unknown function $g$ can be written in the following form.

$$
\begin{aligned}
& \left(h^{* 3}+F_{a}^{*}\right)\left(\frac{\partial^{2} p}{\partial x^{2}}+\frac{\partial^{2} p}{\partial y^{2}}\right)-6 G_{a}^{*} \frac{K^{1 / 2}}{\delta_{r}^{3}}\left(\frac{\partial^{2} g}{\partial x^{2}}+\frac{\partial^{2} g}{\partial y^{2}}\right) \\
& =\frac{12 \mu}{h_{0}^{3}} \frac{d h}{d t} \\
& \left(h^{* 3}+F_{b}^{*}\right)\left(\frac{\partial^{2} p}{\partial x^{2}}+\frac{\partial^{2} p}{\partial y^{2}}\right)-6 G_{b}^{*} \frac{K^{1 / 2}}{\delta_{r}^{3}}\left(\frac{\partial^{2} g}{\partial x^{2}}+\frac{\partial^{2} g}{\partial y^{2}}\right) \\
& =\frac{12}{\delta_{r}^{3} K^{1 / 2}} g+\frac{12 \mu}{h_{0}^{3}} \frac{d h}{d t}
\end{aligned}
$$

where $h_{0}$ denotes the initial film thickness, $K$ is the permeability of porous material, $\delta^{r}=h^{0} / K^{1 / 2}$ and $h^{*}=h /$ $h_{0}$. The expressions $F_{a}^{*}, G_{a}^{*}, F_{b}^{*}$ and $G_{b}^{*}$ are functions of the permeability $K$, the porous-facing thickness $H$, the dimensionless film thickness $h^{*}$ and the parameter $\alpha$, and are given in (5). The parameter $\alpha$ is defined as

$$
\alpha=\left(\frac{\tilde{\mu}}{\mu}\right)^{1 / 2}
$$

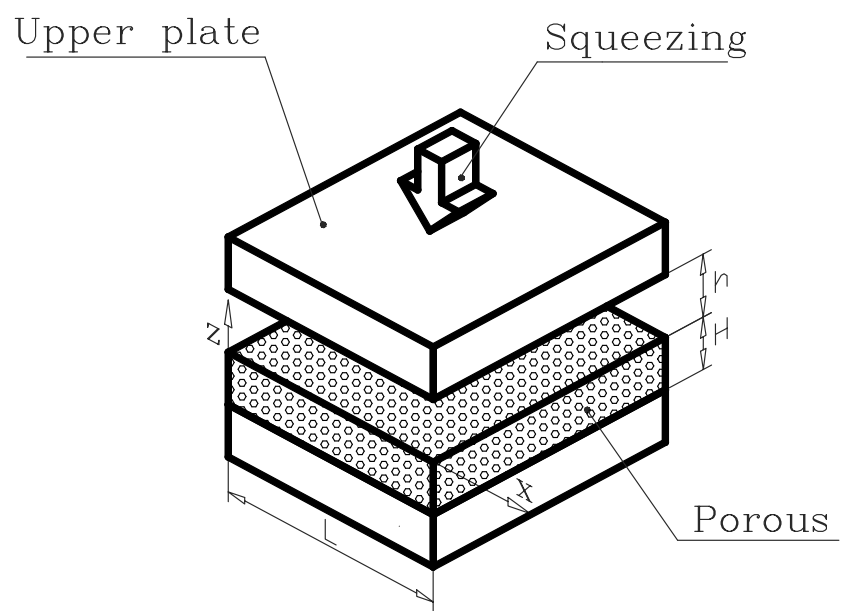

Fig. 1. Squeeze-film mechanism for porous parallel rectangular plates. where $\tilde{\mu}$ represents the effective viscosity of fluid in the permeable matrix which can be assumed to be different in value from $\mu$. Expressed in a dimensionless form the two coupled Reynolds equations are given by

$$
\begin{aligned}
& \left(h^{* 3}+F_{a}^{*}\right)\left(\frac{\partial^{2} p^{*}}{\partial x^{* 2}}+\lambda^{2} \frac{\partial^{2} p^{*}}{\partial y^{* 2}}\right)-\frac{6}{\delta_{r}^{4}} G_{a}^{*}\left(\frac{\partial^{2} g^{*}}{\partial x^{* 2}}+\lambda^{2} \frac{\partial^{2} g^{*}}{\partial y^{* 2}}\right) \\
& =-12 \\
& \left(h^{* 3}+F_{b}^{*}\right)\left(\frac{\partial^{2} p^{*}}{\partial x^{* 2}}+\lambda^{2} \frac{\partial^{2} p^{*}}{\partial y^{* 2}}\right)-\frac{6}{\delta_{r}^{4}} G_{b}^{*}\left(\frac{\partial^{2} g^{*}}{\partial x^{* 2}}+\lambda^{2} \frac{\partial^{2} g^{*}}{\partial y^{* 2}}\right) \\
& =\frac{12}{\delta_{r}^{2} h_{0 L}^{2}}-12
\end{aligned}
$$

where the non-dimensional variables are defined as

$$
\begin{aligned}
& h_{0 L}=\frac{h_{0}}{L}, x^{*}=\frac{x}{L}, y^{*}=\frac{y}{D}, \lambda=\frac{L}{D}, \\
& p^{*}=\frac{p h_{0}^{3}}{\mu L^{2}(-d h / d t)}, g^{*}=\frac{g h_{0}^{4}}{\mu L^{2}(-d h / d t)}
\end{aligned}
$$

Equations (4) and (5) are subject to the following boundary conditions.

$$
\begin{aligned}
& p^{*}=g^{*}=0 \text { at } x^{*}=0 \\
& p^{*}=g^{*}=0 \text { at } x^{*}=1 \\
& p^{*}=g^{*}=0 \text { at } y^{*}=0 \\
& p^{*}=g^{*}=0 \text { at } y^{*}=1
\end{aligned}
$$

Equations (7), (8), (9) and (10) show that the squeeze-film pressure $p^{*}$ at the four ends of the porous parallel rectangular plates are equal to the ambient pressure. Since $p^{*}$ and the pressure in the porous region $\tilde{p}^{*}$ are zero at those ends, one has $g^{*}=0$ at the same boundaries from the analysis in [5].

After eliminating the unknown $p^{*}$ from the coupled Reynolds equations, one gets the partial differential equation for .

$$
\frac{\partial^{2} g^{*}}{\partial x^{* 2}}+\lambda^{2} \frac{\partial^{2} g^{*}}{\partial y^{* 2}}-A g^{*}=B
$$

where

$$
A=2 \frac{\delta_{r}^{2}}{h_{0 L}^{2}} \frac{h^{* 3}+F_{a}^{*}}{G_{a}^{*}\left(h^{* 3}+F_{b}^{*}\right)-G_{b}^{*}\left(h^{* 3}+F_{a}^{*}\right)}
$$




$$
B=2 \frac{\delta_{r}^{4}}{1} \frac{h_{b}^{*}-F_{a}^{*}}{G_{a}^{*}\left(h^{* 3}+F_{b}^{*}\right)-G_{b}^{*}\left(h^{* 3}+F_{a}^{*}\right)}
$$

Using the central difference approximation for the partial derivatives, equations (11) and (4) can be written respectively in a numerical format.

$$
\begin{aligned}
g_{i, j}^{*} & =\gamma_{1}\left(g_{i+1, j}^{*}+g_{i-1, j}^{*}\right)+\gamma_{2}\left(g_{i, j+1}^{*}+g_{i, j-1}^{*}\right)-\gamma_{0} \\
p_{i, j}^{*} & =\alpha_{1}\left(p_{i+1, j}^{*}+p_{i-1, j}^{*}\right)+\alpha_{2}\left(p_{i, j+1}^{*}+p_{i, j-1}^{*}\right)-\alpha_{0} \\
& -\beta_{1}\left(g_{i+1, j}^{*}+g_{i-1, j}^{*}\right)+\beta_{2}\left(g_{i, j+1}^{*}+g_{i, j-1}^{*}\right)+\beta_{3} g_{i, j}^{*}
\end{aligned}
$$

where

$$
\begin{aligned}
& \gamma_{1}=\frac{\delta^{2}}{2 \delta^{2}+2 \lambda^{2}+A\left(\Delta y^{*}\right)^{2}}, \gamma_{2}=\frac{\lambda^{2}}{2 \delta^{2}+2 \lambda^{2}+A\left(\Delta y^{*}\right)^{2}}, \\
& \gamma_{0}=\frac{B\left(\Delta y^{*}\right)^{2}}{2 \delta^{2}+2 \lambda^{2}+A\left(\Delta y^{*}\right)^{2}} \\
& \alpha_{1}=\frac{\delta^{2}}{2\left(\delta^{2}+\lambda^{2}\right)}, \alpha_{2}=\frac{\lambda^{2}}{2\left(\delta^{2}+\lambda^{2}\right)}, \\
& \alpha_{0}=\frac{6\left(\Delta y^{*}\right)^{2}}{\left(\delta^{2}+\lambda^{2}\right)\left(h^{* 3}+F_{a}^{*}\right)} \\
& \beta_{1}=\alpha_{1} \beta_{3}, \beta_{2}=\alpha_{2} \beta_{3}, \beta_{3}=\frac{6 G_{a}^{*}}{\delta_{r}^{4}\left(h^{* 3}+F_{a}^{*}\right)}, \delta=\frac{\Delta y^{*}}{\Delta x^{*}}
\end{aligned}
$$

The mesh for the film region has 20 intervals in the horizontal direction and 20 intervals across the plate width. The finite-difference system of equations (14) and (15) are solved by using the Jacobi method of iteration [4] with the accuracy:

$$
\left|\frac{g_{i, j}^{*(k+1)}-g_{i, j}^{*(k)}}{g_{i, j}^{*(k)}}\right|<0.0001,\left|\frac{p_{i, j}^{*(k+1)}-p_{i, j}^{*(k)}}{p_{i, j}^{*}}\right|<0.0001
$$

where $g_{i, j}^{*(k)}$ and $p_{i, j}^{*(k)}$ denotes the iterative values of $g_{i, j}^{*}$ and $p_{i, j}^{*}$, respectively. Once the film pressure is calculated, the squeeze-film characteristics can be determined.

The load-carrying capacity is obtained by integrating the film pressure over the film region.

$$
W=\int_{y=0}^{D} \int_{x=0}^{L} p d x d y
$$

Expressed in terms of dimensionless quantities and written in a numerical format, one has

$$
W^{*}=\int_{y^{*}=0}^{1} \int_{x^{*}=0}^{1} p^{*} d x^{*} d y^{*} \cong \sum_{i=0}^{M} \sum_{j=0}^{N} p_{i, j}^{*} \Delta x^{*} \Delta y^{*}
$$

The time, taken by the upper plate to move from the $h^{*}=1$ to $h^{*}=h_{1}^{*}$ for a constant load, can be calculated from equation (21). Introduce the non-dimensional time

$$
t^{*}=\frac{W h_{0}^{2}}{\mu L^{3}} t
$$

After rearranging the equation, the time-height relationship is presented in the following form.

$$
\frac{d h^{*}}{d t^{*}}=-\frac{1}{W^{*}} \cong-\frac{1}{\sum_{i=0}^{M} \sum_{j=0}^{N} p_{i, j}^{*} \Delta x^{*} \Delta y^{*}}
$$

This equation is a highly nonlinear equation of first order. The initial condition for the dimensionless film height is:

$$
h^{*}=1 \text { at } t^{*}=0
$$

Applying the fourth-order Runge-Kutta method [4] with a time step of $\Delta t^{*}=0.05$, a numerical solution of the film height is obtained.

\section{RESUYLTS AND DISCUSSION}

To reveal the effects of viscous shear stresses of the BM, the Reynolds equation derived using the SFM and DM by Prakash and Vij [16] is extended to evaluate the squeeze-film pressure and thereafter the squeezefilm characteristics (Appendix A). To illustrate the numerical results, the values of parameters are chosen as: $\lambda=0.1,0.5,1, h_{0 L}=h_{0} / L=0.01, H L=H / L=0.02$. Since from the previous study [5] the effect of variation of $\alpha$ on the one-dimensional porous squeeze-film plates is found to be small and negligible, the value of $\alpha$ in the $\mathrm{BM}$ is similarly chosen to be 1 in evaluating the squeezefilm characteristics of porous parallel rectangular plates.

The comparison of dimensionless squeeze-film pressure $p^{*}$ as a function of dimensionless coordinate $x^{*}$ for different permeability parameter $\Phi$ predicted by the three models is shown in Fig. 2. Comparing with the non-porous plates, the presence of porous-facing material is observed to decrease the squeeze-film pressure. This phenomenon can be explained from the fact that the porous-facing material provides a path for fluid flow toward the environments. When the upper plate approaches the lower one, a part of the fluid is squeezed out. The remaining part will simply flow out through 
the porous material. Therefore, the lubricant becomes easier to flow out of the squeeze-film plates with the porous facing in place. In this sense, the presence of the porous facing decreases the resistance to lubricant flow and makes the film pressure evenly distributed. As shown, an increasing value of $\Phi$ yields a decreasing $p^{*}$. Comparing with the DM, the viscous shear effects of the BM decrease the pressure. But for the artificial SFM, the slip velocity may be over introduced and therefore gives a lower squeeze-film pressure.

The comparison of dimensionless load-carrying capacity $W^{*}$ as a function of permeability parameter $\Phi$ for length-to-width ratio $\lambda=1$ predicted by using the BM, SFM and DM is presented in Fig. 3. Since the presence of the porous facing decreases the squeezefilm pressure, the integrated load-carrying capacity is similarly affected. A decreasing value of $W^{*}$ is obtained with increasing value of $\Phi$. The BM results in a lower load-carrying capacity as compared to that derived by the DM, but the viscous shear effects provide a higher $W^{*}$ as compared to the SFM. Fig. 4 shows the comparison of dimensionless load-carrying capacity $W^{*}$ as a function of permeability parameter $\Phi$ for $\lambda=$ 0.5 and 0.1 predicted by using the BM, SFM and DM. As the plates tend to be wide $(\lambda=0.1)$, a larger amount of the difference of the load-carrying capacity is observed among the three models for moderate values of $\Phi$.

The comparison of time-height relationship for different values of permeability parameter $\Phi$ with lengthto-width ratio $\lambda=1$ predicted by using the BM, SFM and

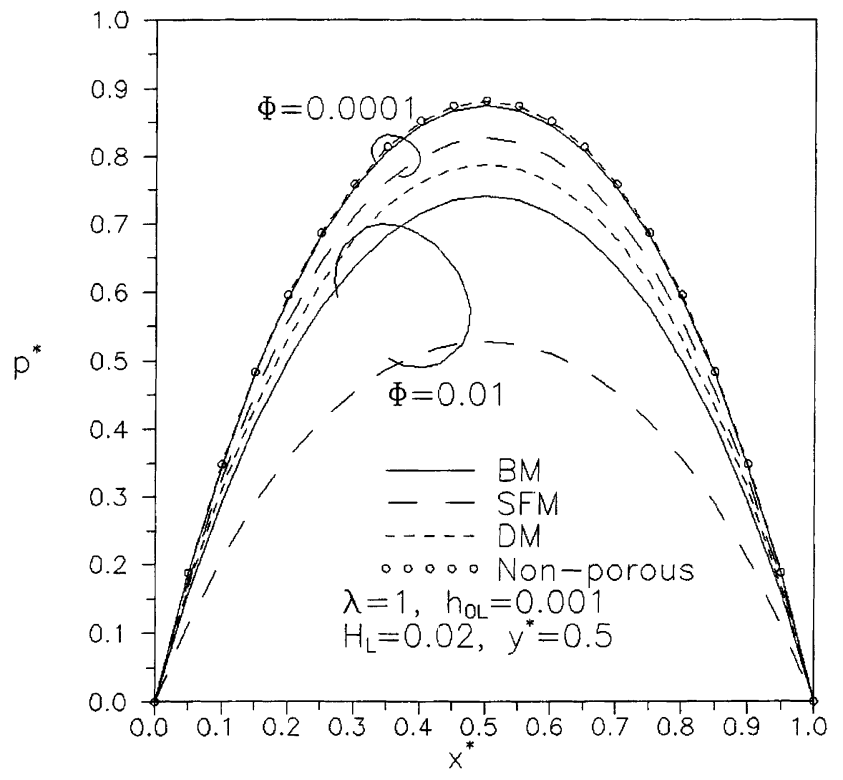

Fig. 2. Comparison of dimensionless squeeze-film pressure $\boldsymbol{p}^{*}$ as a function of dimensionless coordinate $x *$ for different $\Phi$.
DM is shown in Fig. 5. The effects of viscous shear stresses of the $\mathrm{BM}$ reduce the response time as compared to the DM. Comparing with the SFM, the viscous shear effects lengthen the time required to achieve a given film height. Fig. 6 presents the comparison of time-height relationship for different length-to-width ratio $\lambda$ with permeability parameter $\Phi=0.001$ predicted by using the BM, SFM and DM. Since a larger differ-

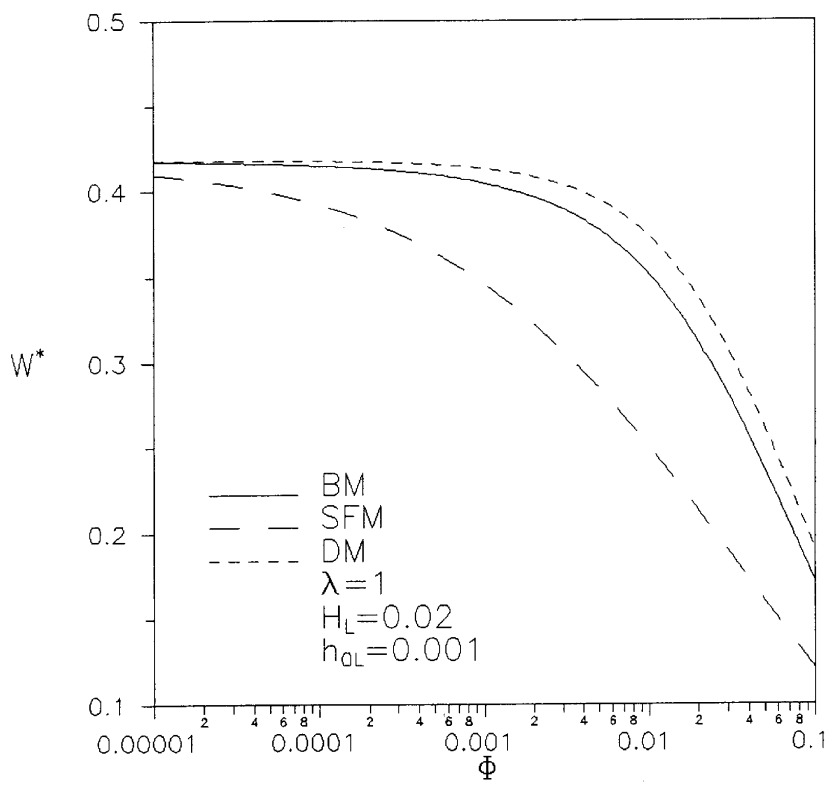

Fig. 3. Comparison of $W^{*}$ as a function of $\Phi$ with $\lambda=1$ between the BM, SFM, and DM.

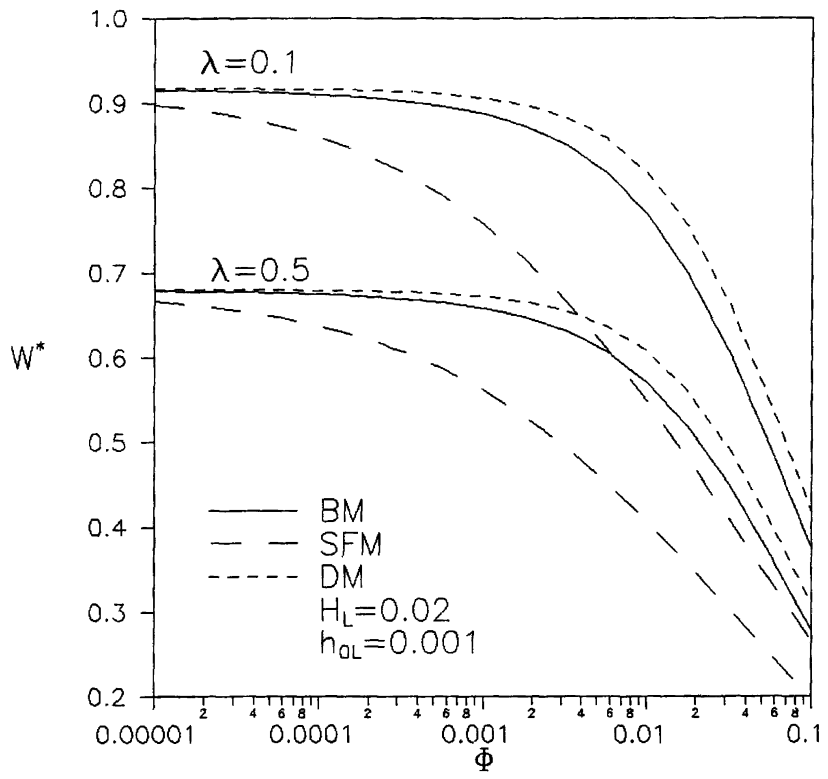

Fig. 4. Comparison of $W^{*}$ as a function of $\Phi$ with $\lambda=0.5$ and 0.1 between the BM, SFM, and DM. 
ence of the load-carrying capacity among the three models is observed when the value of length-to-width is small, it also reflects in the response time. Comparing with the DM and the SFM, the effects of viscous shear stresses of the BM upon the time-height relationship are more pronounced as the plates tend to be wide.

As a design example, we consider the data of porous parallel rectangular plates:

$$
\begin{aligned}
L= & 2.5 \mathrm{~cm} \\
D= & 2.5 ; 5 \mathrm{~cm} \\
H= & 0.025 \mathrm{~cm} \\
h_{0}= & 0.0025 \mathrm{~cm} \\
K= & 6.25 \times 10^{-12} ; 6.25 \times 10^{-11} ; 6.25 \times 10^{-10} ; \\
& 6.25 \times 10^{-9} ; 6.25 \times 10^{-8} \mathrm{~cm}^{2}
\end{aligned}
$$

From these data one has:

$h_{0 L}=\frac{h_{0}}{L}=0.001$

$H_{L}=\frac{H}{L} 0.01$

$\lambda=\frac{L}{D}=1.0 ; 0.5$

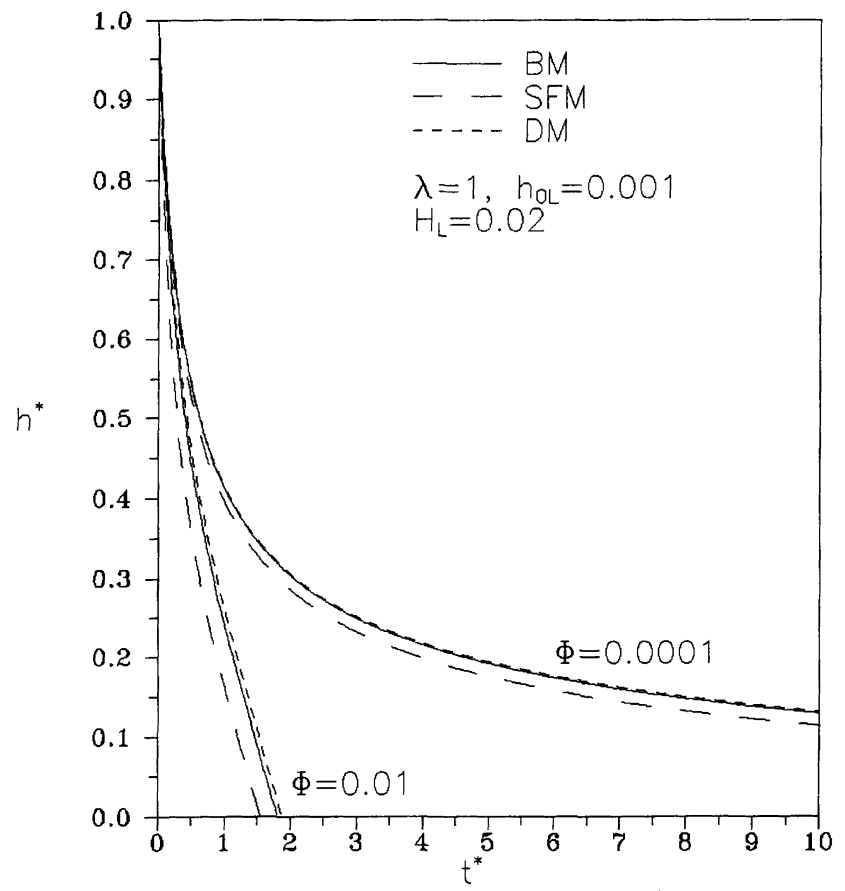

Fig. 5. Comparison of the film thickness $h^{*}$ as a function of $t^{*}$ with $\lambda=$ 1 for the BM, SFM, and DM.

$$
\Phi=\frac{K H}{h_{0}^{3}}=0.00001 ; 0.0001 ; 0.001 ; 0.01 ; 0.1
$$

Following the procedure described in the above analysis, the load-carrying capacity can be evaluated. The results are shown in Table 1 .

\section{CONCLUSIONS}

On the basis of the Brinkman model, the squeezefilm characteristics of two-dimensional porous parallel plates are presented in this paper. Two coupled Reynolds equations derived in a previous study [5] are applied to evaluate the porous squeeze-film behavior. According to the results obtained, the $\mathrm{BM}$ predicts quite different squeeze-film characteristics to those derived by the slip-flow model and the Darcy model. Comparing with the Darcy model, the effects of viscous shear stresses of the Brinkman model decrease the load-carrying capacity and shorten the time required for the upper plate to achieve a given film height. However, the viscous shear effects increase the load-carrying capacity and lengthen the response time of finite porous squeeze-film plates as compared to the slip-flow model. Moreover, the influence of viscous shear stresses of the Brinkman model upon the squeeze-film performance is more pronounced when the plates tend to be wide. A design example is illustrated to demonstrate how the present study could be used for the mechanism of porous parallel plates with

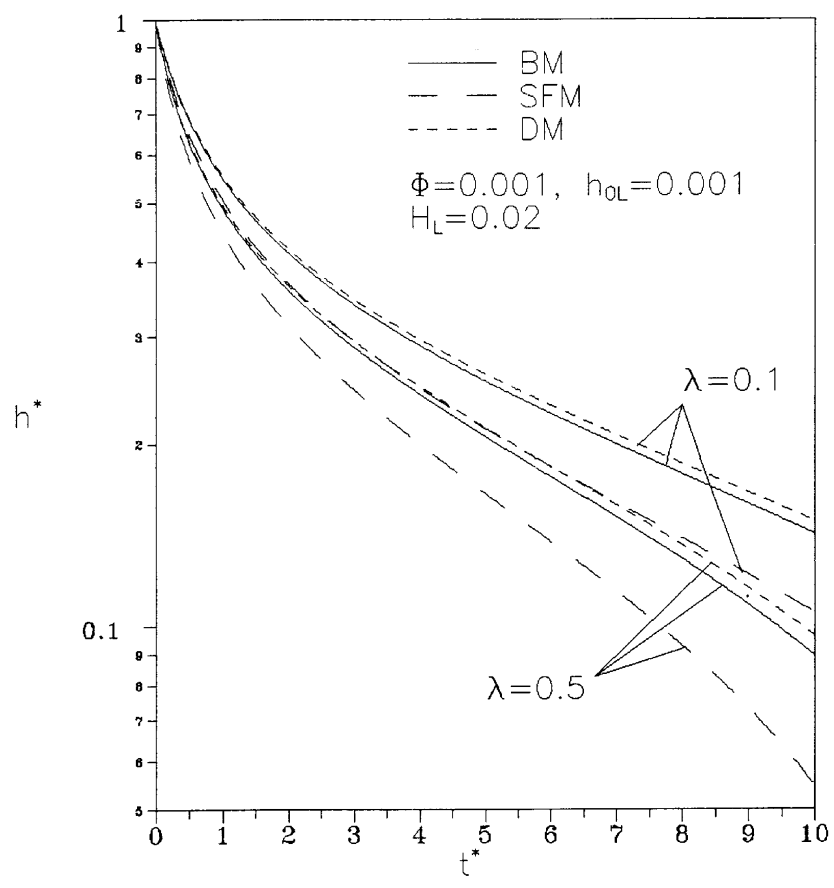

Fig. 6. Comparison of the film thickness $h^{*}$ as a function of $t^{*}$ with $\lambda=$ 0.5 and 0.1 for the BM, SFM, and DM. 
Table 1. Nondimensional load-carrying capacity $\mathrm{W}^{*}$ with $h_{0 L}$ $=0.001$ and $H_{L}=0.01$

\begin{tabular}{ccc}
\hline$\Phi$ & $W^{*}(\lambda=1.0)$ & $W^{*}(\lambda=0.5)$ \\
\hline 0.00001 & 0.417022 & 0.677808 \\
0.00002 & 0.416566 & 0.677066 \\
0.00003 & 0.415940 & 0.676049 \\
0.00006 & 0.415075 & 0.674644 \\
0.00010 & 0.413869 & 0.672682 \\
0.00032 & 0.412168 & 0.669920 \\
0.00056 & 0.409746 & 0.665981 \\
0.00100 & 0.406249 & 0.660295 \\
0.00178 & 0.393583 & 0.651979 \\
0.00316 & 0.382393 & 0.639706 \\
0.00562 & 0.365890 & 0.621513 \\
0.01000 & 0.342039 & 0.594683 \\
0.01778 & 0.308944 & 0.555906 \\
0.03162 & 0.266046 & 0.502105 \\
0.05623 & 0.215566 & 0.432367 \\
0.10000 & 0.162949 & 0.264790 \\
\hline
\end{tabular}

finite width.

\section{ACKNOWLEDGEMENTS}

This work was supported by the National Science Council of ROC: NSC 90-2212-E-253-004.

\section{APPENDIX A: RESULTS PREDICTED BY USING THE SFM AND DM}

The dimensionless Reynolds equation for the twodimensional porous squeeze-film plates has been derived by Prakash and Vij [16].

$$
\frac{\partial^{2} p^{*}}{\partial x^{* 2}}+\lambda^{2} \frac{\partial^{2} p^{*}}{\partial y^{* 2}}=\frac{-12}{h^{* 3}(1+\xi)+12 \Phi}
$$

Where

$$
\xi=\frac{3\left(\delta_{r} h^{*}+2 \alpha_{s}\right)}{\delta_{r} h^{*}\left(\alpha_{s} \delta_{r} h^{*}+1\right)}
$$

In these equations, $\xi$ accounts for the velocity slip and $\alpha_{s}$ denotes a dimensionless slip coefficient. According to Beavers and Joseph [1] and Beavers et al. [2], $\alpha_{s}=0.1$ is used in the SFM. The equation reduces to the no-slip case of the DM as $\xi$ is equal to zero. Following the same procedure as in the present study, one can solve for the squeeze-film pressure and the squeezefilm characteristics of finite porous parallel plates.

\section{NOTATION}

$D$

$g, g^{*}$

$h, h^{*}$

$h_{0}$

$h_{0 L}$

$H$

$H_{L}$

$H_{L}=H / L$

$K \quad$ permeability of porous material

$L \quad$ length of the porous plate

$p, p^{*} \quad$ squeeze-film pressure, $p^{*}=p h_{0}^{3} / \mu L^{2}(-d h / d t)$

(nondimensional)

$t, t^{*}$

$u, v, w$

$W, W^{*}$

$x, y, z$

$x^{*}, y^{*}$

$\alpha$

$\lambda$

$\delta_{r}$

$\mu$

$\underset{\sim}{\Phi}$

$\tilde{()}$

width of the porous plate

pressure gradient across the porous medium, $g^{*}=g h_{0}^{4} / \mu L^{2}(-d h / d t)$ (nondimensional)

film thickness, $h^{*}=h / h_{0}$ (nondimensional)

initial film thickness

$h_{0 L}=h_{0} / L$

wall thickness of porous surface

time $\mathrm{t}^{*}=W h_{0}^{2} t / \mu L^{3}$ (nondimensional)

velocity components in the film region

load-carrying capacity, $W^{*}=W h_{0}^{3} / \mu L^{3} D(-d h /$

$d t$ ) (nondimensional)

rectangular coordinate

dimensionless coordinates, $x^{*}=x / L, y^{*}=y / D$ $\alpha=(\tilde{\mu} / \mu)^{1 / 2}$

length-to-width ratio, $\lambda=L / D$

$\delta_{r}=h_{0} / K^{1 / 2}$

lubricant viscosity

permeability parameter, $\Phi=K H / h_{0}^{3}$

quantities within the porous medium

\section{REFERENCE}

1. Beavers, G.S. and Joseph, D.D., "Boundary Conditions at a Naturally Permeable Wall," J. Fluid Mechanics, Vol. 30, No. 1, pp. 191-207 (1967).

2. Beavers, G.S., Sparrow, EM., and Magnuson, RA., "Experiments on Coupled Parallel Flows in a Channel and a Bounding Porous Medium," J. Basic Engineering, Vol. 92, No. 4, pp. 843-848 (1970).

3. Elsharkawy, A.A. and Nassar, M.N.,"Hydrodynamic Lubrication of Squeeze-Film Porous Bearing," Acta Mechanica, Vol. 118, pp. 121-134 (1996).

4. Faires, J.D. and Burden, R., Numerical Methods, Brooks/ Cole Publishing Company, Pacific Grove, p. 303 (1998).

5. Lin, J.R., "Derivation of Porous Squeeze-film Reynolds Equations Using the Brinkman Model and its Application," J. Physics D: Appl. Phys., Vol. 34, pp. 3217-3233 (2001).

6. Lin, J.R., "Dynamic Behavior of Pure Squeeze Films in Short Porous Journal Bearings Using the Brinkman Model," J. Physics D: Appl. Phys., Vol. 28, pp. 21882196 (1995).

7. Lin, J.R., "Pure Squeeze Film Behavior in a Hemispherical Porous Bearing Using the Brinkman Model," STLE Tribol. Trans., Vol. 39, No. 4, 769-778 (1996).

8. Lin, J.R., "Viscous Shear Effects on the Squeeze-Film 
Behavior in Porous Circular Disks," Int. J. Mech. Sci., Vol. 39, No. 4, pp. 373-384 (1996).

9. Megat Ahmad, M.M.H., Gethin, D.T., Claypole, T.C., and Roylance, B.J., "Numerical and Experimental Investigation into Porous Squeeze Films," Tribol. Int., Vol. 31, No. 4, pp. 189-199 (1998).

10. Murti, P.R.K., "Squeeze Film Behavior in a Spherical Porous Bearing," ASME J. Lubr. Technol., Vol. 97, pp. 638-641 (1975).

11. Murti, P.R.K., "Squeeze Films in Porous Circular Disks," Wear, Vol. 23, pp. 283-289 (1973).

12. Murti, P.R.K., "Squeeze-Film Behavior in Porous Circular Disks," ASME J. Lubr. Technol., Vol. 96, pp. 206209 (1974).

13. Naduvinamani, N.B., Hiremath, P.S. and Gurubasavaraj, G., "Static and Dynamic Behaviour of Squeeze-Film Lubrication of Narrow Porous Journal Bearings with Coupled Stress Fluid," Proc. Instn. Mech. Engrs., Vol. 215(J), pp. 45-62 (2001).

14. Neale, G. and Nader, W., "Practical Significance of Brinkman's Extension of Darcy's Law: Coupled Parallel Flows within a Channel and a Bounding Porous
Medium," Can. J. Chem. Engin., Vol. 52, pp. 475-478 (1974).

15. Prakash, J. and Vij, S.K., "Squeeze Films in Porous Bearing,"Wear, Vol. 27, pp. 359-366 (1974).

16. Prakash, J., Vij, SK, "Effect of Velocity Slip on PorousWalled Squeeze Films," Wear, Vol. 29, pp. 363-372 (1974).

17. Sparrow, E.M., Beavers, G.S., and Hwang, I.T., "Effect of Velocity Slip on Porous-Walled Squeeze Films," ASME J. Lubr. Technol., Vol. 94, pp. 260-265 (1972).

18. Wu, H., "An Analysis of the Squeeze Film Between Porous Rectangular Plates," ASME J. Lubr. Technol., Vol. 94, pp. 64-68 (1972).

19. Wu, H., "Effect of Velocity-slip on the Squeeze Film Between Porous Rectangular Plates," Wear, Vol. 20, pp. 67-71 (1972).

20. Wu, H., "Squeeze-film Behavior for Porous Annular Disks," ASME J. Lubr. Technol., Vol. 92, pp. 593-596 (1970).

21. Wu, H., "The Squeeze Film Between Rotating Porous Annular Disks,” Wear, Vol. 18, pp. 461-470 (1971). 